CLINICAL STUDY

\title{
Low testosterone and sex hormone-binding globulin levels and high estradiol levels are independent predictors of type 2 diabetes in men
}

\author{
Torkel Vikan ${ }^{1,4}$, Henrik Schirmer ${ }^{2,3}$, Inger Njølstad ${ }^{3}$ and Johan Svartberg ${ }^{1,4}$ \\ ${ }^{1}$ Division of Internal Medicine and ${ }^{2}$ Department of Cardiology, University Hospital of North Norway, Tromso 9038, Norway, ${ }^{3}$ Institute of Community \\ Medicine and ${ }^{4}$ Institute of Clinical Medicine, University of Tromsø, Tromsø 9037, Norway
}

(Correspondence should be addressed to T Vikan at Division of Internal Medicine, University Hospital of North Norway; Email: torkel.vikan@unn.no)

\begin{abstract}
Objective: To study the impact of endogenous sex hormone levels in community-dwelling men on later risk for type 2 diabetes.

Design: Population-based prospective cohort study.

Methods: For the analyses, 1454 men who participated in the fourth Tromsø study (1994-1995) were used. Cases of diabetes were retrieved and validated until 31.12.05 following a detailed protocol. The prospective association between sex hormones and diabetes was examined using Cox proportional hazard regression analysis, allowing for multivariate adjustments.

Results: There was a significantly lowered multi-adjusted risk for later diabetes with higher normal total testosterone levels, both linearly per s.D. increase (hazard ratio (HR) 0.71, confidence interval (CI) 0.54-0.92) and in the higher quartiles of total testosterone than in the lowest quartiles (HR 0.53, CI 0.33-0.84). A reduced multi-adjusted risk for incident diabetes was also found for men with higher sex hormone-binding globulin (SHBG) levels, both linearly per s.D. increase (HR 0.55, CI 0.39-0.79) and when comparing the third (HR 0.38, CI 0.18-0.81) and the fourth quartile (HR 0.37, CI 0.17-0.82) to the lowest quartile. The associations with total testosterone and SHBG were no longer significant after inclusion of waist circumference to the multivariate models. Estradiol $\left(\mathrm{E}_{2}\right)$ was positively associated with incident diabetes after multivariate adjustments including waist circumference when comparing the second (HR 0.49, CI 0.26-0.93) and the third (HR 0.51, CI 0.27-0.96) quartile to the highest quartile.

Conclusion: Men with higher $\mathrm{E}_{2}$ levels had an increased risk of later diabetes independent of obesity, while men with lower total testosterone and SHBG had an increased risk of diabetes that appeared to be dependent on obesity.
\end{abstract}

European Journal of Endocrinology 162 747-754

\section{Introduction}

Hypogonadism of aging males and the metabolic syndrome share many common traits as lower testosterone levels have been associated with abdominal obesity (1), hypertension (2), higher glucose and insulin (3), and dyslipidemia (4). Furthermore, men with type 2 diabetes mellitus (for simplicity only the term diabetes is used in the following paragraphs) have a high prevalence of hypogonadotropic hypogonadism (5). In a cross-sectional study of men who participated in the Tromsø study, both lower total testosterone and sex hormone-binding globulin (SHBG) levels were associated with increased $\mathrm{HbAlc}$ levels, independently of obesity (6), and some prospective observational studies have given support for a predictive role of both lower testosterone and SHBG levels in later diabetes $(7,8)$.
Intervention studies have reported improvements in body composition (9) and insulin sensitivity (10) with testosterone supplementation. In hypogonadal men over the age of 30 years with diabetes, testosterone replacement led to improvements in insulin resistance, glycemic control, and visceral obesity $(11,12)$, while acute withdrawal of testosterone supplementation in men with idiopathic hypogonadotropic hypogonadism led to a worsening of insulin sensitivity, without detectable changes in body fat content (13). By contrast, in a randomized study of recombinant human chorionic gonadotropin treatment of older men, no change in the measures of insulin sensitivity was found in the treatment group, in spite of improvements in body composition (14). Another study suggested that lower testosterone levels may promote insulin resistance through impairment of mitochondrial function (15). Thus, as to whether male hypogonadism plays a causal 
role in the development of insulin resistance and diabetes or is a consequence of it, and to which mechanisms a possible preventive effect of conserved testosterone levels may be ascribed to need to be investigated further.

We aimed to study the impact of sex hormone levels in men on the risk of subsequent diabetes in a population of 1454 community-dwelling men with 76 cases of incident diabetes. To our knowledge, this is the largest prospective study to date addressing this issue.

\section{Materials and methods}

\section{Study population}

The Tromsø study is a population-based prospective study with repeated health surveys, primarily focusing on cardiovascular and other chronic diseases. The population of the fourth Tromsø study (1994-1995) has been described previously in detail (16). From the fourth Tromsø study, data on sex hormones were available in a randomly selected sub-sample of 1579 men. Eleven men were excluded because of testosterone levels suggestive of medical or surgical castration. Another 114 men were excluded from the dataset for the following reasons: 51 men who answered 'yes' to the question 'do you have, or have you had diabetes?' at the baseline questionnaire, ten men who had HbAlc levels $>6.5 \%$ at baseline, 11 men with unknown date for the diagnosis of diabetes, eight men who got a validated diagnosis of diabetes the same year as the baseline examination or earlier, ten men who moved from the municipality before the year of the baseline examination, and 24 men with a diagnosis of diabetes after 2005 (after which the diabetes endpoint is not validated). One thousand four hundred and fifty-four men remained for analysis of the impact of sex hormones on the incidence of type 2 diabetes.

\section{Definition and ascertainment of type 2 diabetes}

A committee validated the diabetes endpoint. Possible cases were retrieved either by self-report at the sixth Tromsø study (2007-2008), in which 11000 of the 27158 participants of the fourth Tromsø study attended, by the linkage of the fourth Tromsø study participant list to the University Hospital of North Norway digital discharge diagnosis registry (ICD-9 codes 250, 357.2, 362.0, 583.8, 648.0, 648.8, 790.2, ICD-10 codes E10.0-E14, O24 and R73) in conjunction with a concurrent cardiovascular disease (CVD) registration (if the CVD adjudication procedure revealed that the patients also had diabetes), or by linkage to the Causes of Death Registry. Cases of diabetes registered by means of one of these procedures were verified by medical record information at the University Hospital of North Norway, and were recorded if diabetes was mentioned in the hospital record notes or in the referral letter(s) from physicians. Cases of unknown type of diabetes were not included in the analyses, and only cases of verified type 2 diabetes were used. Complete assessment of the endpoint was done until 31.12.05. Information on death, migration from the municipality, and emigration from Norway was obtained from the Norwegian Registry of Vital Statistics.

\section{Baseline characteristics}

Height and weight were measured with subjects standing wearing light clothing without shoes. Waist circumference was measured at the umbilical line according to a written protocol. Body mass index $\left(\mathrm{BMI} ; \mathrm{kg} / \mathrm{m}^{2}\right)$ was calculated. Blood pressure was recorded three times with an automatic device (Dinamap Vital Signs Monitor, Critikon Inc., Tampa, FL, USA) by specially trained personnel, and the mean of readings 2 and 3 was used in the analysis. Non-fasting blood samples were drawn between 0800 and $1600 \mathrm{~h}$. Serum total cholesterol and triglycerides were analyzed by enzymatic colorimetric methods with commercial kits (CHOD-PAP for cholesterol and GPO-PAP for triglycerides; Boehringer-Mannheim). Serum highdensity lipoprotein (HDL) cholesterol was measured after precipitation of lower density lipoprotein with heparin and manganese chloride. Self-administered questionnaires that included information about the history of coronary heart disease (CHD), CVD, smoking habits, physical activity, and medical history were checked by trained nurses.

\section{Sex hormones}

Serum samples obtained in 1994 were analyzed for sex hormones in the autumn of 2001. All samples were stored frozen at $-70{ }^{\circ} \mathrm{C}$ until they were first thawed in 2001 . The determination of total testosterone, estradiol $\left(\mathrm{E}_{2}\right)$, and SHBG was done on Immulite 2000 (Diagnostic Product Corp., Los Angeles, CA, USA). The intra- and interassay coefficients of variation for the analyses were between 5 and $10 \%$. Free testosterone values were calculated from total testosterone and SHBG using a fixed albumin concentration according to Vermeulen et al. (17).

\section{Statistical analysis}

Normal distribution was assessed by skewness and histograms. $\mathrm{E}_{2}$ was not normally distributed, but after logarithmic transformation, $\mathrm{E}_{2}$ assumed normal distribution. In analyses using $E_{2}$ as a continuous variable, the logged value was used. Associations between total and free testosterone and baseline characteristics were examined using age-adjusted partial correlation analysis. 
Differences in baseline characteristics between those who were diagnosed with diabetes during follow-up and those who were not were tested by independent samples $t$-test for continuous variables and by the $\chi^{2}$ test for dichotomous variables. The prospective associations of sex hormones and SHBG (for simplicity only the term sex hormones is used in the following paragraphs) with incident diabetes were examined using Cox proportional hazard regression analysis. Sex hormones were used both as continuous variables with hazard ratios (HRs) for 1 s.D. increase in hormone level, and as quartiles based on the entire population sample. The lowest quartile was used as the reference category for total and free testosterone and SHBG, while the highest quartile was used as the reference category for $\mathrm{E}_{2}$. Total testosterone and free testosterone were also examined as dichotomous variables using the lowest quartile as the reference category. To explore the relative merits of total testosterone and SHBG in predicting diabetes, we repeated the analyses with the inclusion of both the variables in the same model. For all regression analyses, two models were evaluated: one with multivariate adjustment for age, HDL-cholesterol, and systolic blood pressure, and another with the addition of waist circumference to the first model. Only baseline variables associated with both total testosterone and incident diabetes were included in the multivariate models as possible confounders, and baseline variables were excluded if they lost their significant contribution when entering the multivariate models.

The proportional odds assumption was examined using log-minus-log plots, and the assumptions were considered met by all the models. A $P$ value $<0.05$ was considered statistically significant, and all $P$ values that are presented are two-tailed. The SPSS statistical software version 15.0 SPSS Inc., Chicago, IL, USA for Windows was used for all analyses.

\section{Ethics}

The study protocol was approved by the Regional Committee for Medical and Health Research Ethics, North Norway, and informed written consent was obtained from all participants.

\section{Results}

\section{Sex hormones and baseline characteristics}

Baseline characteristics and age-adjusted partial correlations with total and free testosterone and $E_{2}$ are presented in Table 1. Both total testosterone and free testosterone were inversely correlated with age, but the correlation with free testosterone was stronger than that with total testosterone. Total testosterone was inversely associated with waist circumference, BMI, triglycerides, and systolic and diastolic blood pressure, and was positively associated with HDL-cholesterol, $\mathrm{SHBG}, \mathrm{E}_{2}$, and smoking. Free testosterone was also inversely associated with waist circumference, BMI, and systolic blood pressure, and was positively associated with smoking, but the associations were in general weaker than those for total testosterone. Total or free testosterone did not differ between men with or without a history of CHD and/or CVD or in men with less physical activity compared with those with more vigorous physical activity. $\mathrm{E}_{2}$ correlated negatively only with total cholesterol, and positively only with age and total and free testosterone.

Table 1 Baseline characteristics of the 1454 men who participated in the Tromsø study, 1994-1995. Age-adjusted correlations with testosterone, free testosterone, and estradiol $\left(\mathrm{E}_{2}\right)$.

\begin{tabular}{|c|c|c|c|c|}
\hline & Mean or percentage (s.D.) & Total testosterone $(R)$ & Free testosterone $(R)$ & Estradiol $\left(R^{\mathrm{a}}\right)$ \\
\hline Age (years) & $59.4(10.3)$ & $-0.10^{\star}$ & $-0.43^{\star}$ & $0.13^{*}$ \\
\hline Waist $(\mathrm{cm})$ & $95.0(9.0)$ & $-0.34^{*}$ & $-0.09^{*}$ & -0.01 \\
\hline BMI & $26.0(3.4)$ & $-0.31^{\star}$ & $-0.07^{\dagger}$ & -0.01 \\
\hline Total cholesterol $(\mathrm{mmol} / \mathrm{l})$ & $6.5(1.2)$ & 0.05 & $0.08^{\dagger}$ & $-0.10^{\dagger}$ \\
\hline HDL-cholesterol (mmol/l) & $1.39(0.39)$ & $0.11^{\star}$ & -0.04 & -0.03 \\
\hline Triglycerides $(\mathrm{mmol} / \mathrm{l})^{\mathrm{b}}$ & $1.6(0.9)$ & $-0.19^{*}$ & $<0.01$ & -0.06 \\
\hline HbAlc (\%) & $5.36(0.38)$ & -0.02 & $<0.01$ & -0.01 \\
\hline Total testosterone $(\mathrm{nmol} / \mathrm{l})$ & $13.4(5.2)$ & - & $0.73^{*}$ & $0.21^{\star}$ \\
\hline SHBG $(\mathrm{nmol} / \mathrm{l})$ & $52.7(23.6)$ & $0.63^{*}$ & 0.02 & 0.04 \\
\hline Free testosterone $(\mathrm{pmol} / \mathrm{l})$ & $206.4(75.6)$ & $0.73^{*}$ & - & $0.18^{\star}$ \\
\hline Estradiol $(\mathrm{nmol} / /)^{\mathrm{b}}$ & $0.060(0.031)$ & $0.21^{*}$ & $0.18^{*}$ & - \\
\hline Systolic blood pressure $(\mathrm{mmHg})$ & $140.6(19.8)$ & $-0.12^{*}$ & $-0.06^{\dagger}$ & $<0.01$ \\
\hline $\begin{array}{l}\text { Diastolic blood pressure } \\
(\mathrm{mmHg})\end{array}$ & $81.6(11.6)$ & $-0.09^{\star}$ & -0.03 & -0.01 \\
\hline $\mathrm{CHD}(\%)$ & 14.3 & -0.02 & 0.05 & 0.01 \\
\hline CVD (\%) & 15.8 & -0.04 & 0.03 & $<0.01$ \\
\hline Current smoking (\%) & 33.8 & $0.24^{\star}$ & $0.13^{*}$ & $<0.01$ \\
\hline Vigorous physical activity (\%) & 11.4 & 0.01 & -0.01 & $<0.01$ \\
\hline
\end{tabular}

${ }^{\star} P<0.001 ;{ }^{\dagger} P<0.01 . R=$ correlation coefficient.

${ }^{\mathrm{a}} \mathrm{Log}$ value was used for analysis. 
Table 2 Differences in baseline characteristics between the 76 men who did and the 1378 men did not develop diabetes.

\begin{tabular}{lccc}
\hline & & $\begin{array}{c}\text { No } \\
\text { diabetes }\end{array}$ & $\boldsymbol{P}^{\mathrm{a}}$ \\
& Diabetes & & 0.313 \\
Age (years) & 60.6 & 59.4 & $<0.001$ \\
Waist (cm) & 102.4 & 94.5 & $<0.001$ \\
BMI & 28.5 & 25.9 & 0.08 \\
Total cholesterol (mmol/l) & 6.78 & 6.52 & $<0.001$ \\
HDL-cholesterol (mmol/l) & 1.22 & 1.40 & $<0.001$ \\
Triglycerides (mmol//) & 2.01 & 1.58 & $<0.001$ \\
HbAlc (\%) & 5.70 & 5.34 & $<0.001$ \\
Total testosterone (nmol/l) & 11.3 & 13.6 & $<0.001$ \\
SHBG (nmol/l) & 42.0 & 53.3 & 0.782 \\
Free testosterone (pmol/l) & 204.1 & 206.5 & 0.046 \\
Estradiol (nmol//) & 0.07 & 0.06 & $<0.001$ \\
Systolic blood pressure & 148.8 & 140.1 & \\
$\quad$ (mmHg) & & & \\
Diastolic blood pressure & 85.9 & 81.3 & 0.001 \\
$\quad$ (mmHg) & & & \\
CHD (\%) & 22.7 & 13.8 & 0.032 \\
CVD (\%) & 25.3 & 15.3 & 0.020 \\
Current smoking (\%) & 30.3 & 34.0 & 0.499 \\
Vigorous physical activity & 4.0 & 11.8 & 0.038 \\
$\quad(>3$ h/week, \%) & & & \\
\hline
\end{tabular}

${ }^{\text {a }}$ Tested by independent samples $t$-test for continuous variables and by Pearson $\chi^{2}$ test for dichotomous variables.

${ }^{\mathrm{b}}$ The log value was used for analysis.

\section{Baseline characteristics and diabetes}

Baseline characteristics of those who did and did not develop diabetes during the follow-up are presented in Table 2. Those who developed diabetes had significantly higher mean waist circumference, BMI, triglycerides, HbAlc, $\mathrm{E}_{2}$, and systolic and diastolic blood pressure, and a higher proportion of men had CHD and/or CVD. The mean total testosterone, SHBG, and HDL-cholesterol levels were lower, and the proportion of men spending more than $3 \mathrm{~h} /$ week on vigorous physical activity was lower among men who later developed diabetes than among those who did not develop diabetes.

\section{Sex hormones and diabetes}

During follow-up, 93 men were censored due to migration or emigration from the area and 290 men died. Seventy-six men got a diagnosis of diabetes during a mean follow-up of 9.1 years (13 175 person-years), corresponding to an incidence rate of 5.8 per 1000 person-years (Fig. 1).

Adjusted HRs for diabetes by 1 s.D. increase in endogenous sex hormone level are presented in Table 3. There was a significant decrease in risk for diabetes by 1 s.D. increase in total testosterone, HR 0.71 (95\% confidence interval (CI) $0.54-0.92$ ), and by 1 s.D. increase in SHBG, HR 0.55 (95\% CI 0.39-0.79), after adjustment for age, HDL-cholesterol, and systolic blood pressure. When adding waist circumference, model 2, the reduced risk for diabetes by 1 s.D. increase in total testosterone and SHBG was no longer significant.

Table 4 shows the adjusted HRs for diabetes across quartiles of hormone levels. For total testosterone, there was a decrease in the risk of diabetes when comparing the highest quartile with the lowest quartile, HR 0.41 (95\% CI 0.20-0.84), after adjustment for age, systolic blood pressure, and HDL-cholesterol, but not after adding waist circumference to the model. There was a significant decrease in the risk of diabetes, HR 0.42 (95\% CI 0.20-0.89), when comparing the third quartile of free testosterone with the lowest quartile, after multivariate adjustments, but not when including waist circumference. As for SHBG, there was a decrease in the risk of diabetes in both the third, HR 0.38 (95\% CI 0.18-0.81), and the fourth quartile, HR 0.37 (95\% CI $0.17-0.82)$, than in the lowest quartile in the multivariate analysis; however, no significant risk was observed after adding waist circumference. When dichotomized, there was a reduction in risk for diabetes for men in the higher quartiles of total testosterone than for those in the lowest quartile, HR 0.53 (0.33-0.84), but this was no longer significant when adding waist circumference (data not shown). When including total testosterone and SHBG in the same multivariate model, none of them contributed significantly when modeled as quartiles when comparing the lowest quartiles with the higher quartiles. When modeled as continuous variables with HR per S.D. increase, there was still a predictive effect of SHBG (HR 0.58, CI 0.37-0.92) but not of total testosterone (HR 0.94, CI 0.66-1.33) when included in the same multivariate model before but not after adjustment for waist circumference (data not shown). The correlation between total testosterone and SHBG is strong $(r=0.63)$.

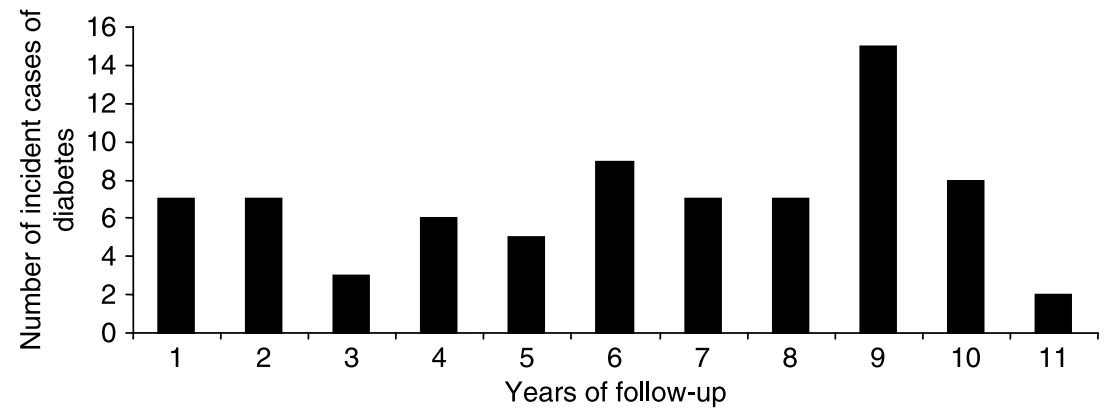

Figure 1 Number of incident cases of diabetes by years of follow-up.

www.eje-online.org 
Table 3 Adjusted hazard ratios (HRs) for incident diabetes by 1 s.D. increase in sex hormone levels in the Tromsø study, $1994-2005$.

\begin{tabular}{lccc}
\hline & Mean hormone level & Model 1 ${ }^{\mathrm{a}}$ HR $(95 \% \mathrm{Cl})$ & Model 2 $^{\mathrm{b}}$ HR $(95 \% \mathrm{Cl})$ \\
\hline Total testosterone $(\mathrm{nmol} / \mathrm{l})$ & 13.4 & $0.71(0.54-0.92)$ & $0.87(0.66-1.15)$ \\
SHBG (nmol/l) & 52.7 & $0.55(0.39-0.79)$ & $0.74(0.51-1.06)$ \\
Free testosterone $(\mathrm{pmol} / \mathrm{l})$ & 206.4 & $1.04(0.80-1.35)$ & $1.15(0.88-1.49)$ \\
${\text { Estradiol }(\mathrm{nmol} /)^{\mathrm{c}}}$ & 0.060 & $1.20(0.92-1.56)$ & $1.24(0.94-1.64)$ \\
\hline
\end{tabular}

${ }^{a}$ Adjusted for age, HDL-cholesterol, and systolic blood pressure.

${ }^{b}$ Adjusted for age, HDL-cholesterol, systolic blood pressure, and waist circumference.

${ }^{\mathrm{c}} \mathrm{Hazard}$ ratios are for 1 S.D. increase in the log value.

As seen in Table 4, there was no significant change in risk for diabetes across the quartiles of $\mathrm{E}_{2}$ when adjusting for age, systolic blood pressure, and HDLcholesterol, but when adding waist circumference to the model, there was a reduction in risk when comparing the second quartile, HR 0.49 (95\% CI 0.26-0.93), and third quartile, HR 0.51 (95\% CI 0.27-0.96), with the highest quartile.

Adjusting for sampling time in the analyses did not materially change the result (data not shown).

\section{Discussion}

In this prospective study of 1454 community-dwelling men, we found a significantly lowered risk for later diabetes with higher normal total testosterone levels, both linearly per S.D. increase (HR 0.71, CI 0.54-0.92) and in the higher quartiles of total testosterone than in the lowest quartiles (HR 0.53, CI 0.33-0.84). A reduced risk for incident diabetes was found for men with higher SHBG levels, both linearly per s.D. increase (HR 0.55, CI 0.39-0.79) and when comparing the third (HR 0.38, CI 0.18-0.81) and the fourth quartile (HR 0.37, CI 0.17-0.82) to the lowest quartile. Associations with total testosterone and SHBG were not independent of waist circumference. By contrast, $\mathrm{E}_{2}$ was positively associated with incident diabetes after multivariate adjustments including waist circumference when comparing the second (HR 0.49, CI 0.26-0.93) and the third (HR 0.51, CI 0.27-0.96) quartile to the highest quartile.

The loss of significant contribution from total testosterone when adding waist circumference to the model may indicate that a protective effect of higher normal testosterone levels is mediated through its effect on central obesity (18), or alternatively, that waist circumference act as a confounder in this setting. Laaksonen et al. (7) studied 702 men with 57 cases of diabetes during 11 years, and found an increased risk of incident diabetes with total testosterone levels in the lowest quartile after multivariate adjustment (odds ratio (OR) 2.37, CI 1.32-4.24) that remained significant but with a weaker risk estimate after inclusion of waist/hip ratio in the model (OR 1.95, CI 1.08-3.55), indicating at least a contributing role for abdominal

Table 4 Adjusted hazard ratios (HRs) for incident diabetes by quartiles of sex hormone levels in the Tromsø study, 1994-2005.

\begin{tabular}{|c|c|c|c|c|}
\hline Hormone & Events & $\begin{array}{c}\text { Mean } \\
\text { hormone level }\end{array}$ & $\begin{array}{c}\text { Model } 1^{\mathrm{a}} \text { HR } \\
(95 \% \mathrm{Cl})\end{array}$ & $\begin{array}{c}\text { Model 2 }{ }^{\mathrm{b}} \text { HR } \\
(95 \% \mathrm{Cl})\end{array}$ \\
\hline \multicolumn{5}{|c|}{ Total testosterone $(\mathrm{nmol} / \mathrm{l})$} \\
\hline Q1 $(<9.9)$ & 33 & 7.6 & 1.0 (Ref) & 1.0 (Ref) \\
\hline Q2 (9.9-12.9) & 18 & 11.4 & $0.58(0.32-1.05)$ & $0.70(0.38-1.26)$ \\
\hline Q3 (12.9-16.2) & 15 & 14.4 & $0.57(0.31-1.06)$ & $0.75(0.40-1.41)$ \\
\hline Q4 (>16.2) & 10 & 20.3 & $0.41(0.20-0.84)$ & $0.67(0.32-1.42)$ \\
\hline Q1 $(<159)$ & 24 & 124 & 1.0 (Ref) & 1.0 (Ref) \\
\hline Q2 (159-197) & 19 & 178 & $0.76(0.41-1.41)$ & $0.91(0.49-1.70)$ \\
\hline Q3 (197-243) & 11 & 219 & $0.42(0.20-0.89)$ & $0.50(0.23-1.05)$ \\
\hline Q4 (>243) & 22 & 304 & $1.05(0.56-1.96)$ & 1.29 (0.69-2.43) \\
\hline \multicolumn{5}{|l|}{ SHBG $(\mathrm{nmol} / \mathrm{l})$} \\
\hline Q1 $(<37.0)$ & 29 & 28.6 & 1.0 (Ref) & 1.0 (Ref) \\
\hline Q2 (37.0-48.0) & 28 & 42.7 & $0.88(0.51-1.53)$ & $1.05(0.60-1.83)$ \\
\hline Q3 (48.0-63.0) & 10 & 55.5 & $0.38(0.18-0.81)$ & $0.56(0.26-1.21)$ \\
\hline \multicolumn{5}{|l|}{ Estradiol (nmol/l) } \\
\hline Q1 $(<0.04)$ & 16 & 0.021 & $0.71(0.38-1.32)$ & $0.65(0.35-1.22)$ \\
\hline Q2 (0.04-0.06) & 15 & 0.046 & 0.57 (0.30-1.08) & $0.49(0.26-0.93)$ \\
\hline Q3 (0.06-0.08) & 14 & 0.065 & $0.55(0.29-1.04)$ & $0.51(0.27-0.96)$ \\
\hline Q4 (>0.08) & 31 & 0.098 & 1.0 (Ref) & 1.0 (Ref) \\
\hline
\end{tabular}

${ }^{a}$ Adjusted for age, HDL-cholesterol, and systolic blood pressure.

${ }^{\mathrm{b}}$ Adjusted for age, HDL-cholesterol, systolic blood pressure, and waist circumference. 
obesity. In a study of 1156 men, of whom 54 developed diabetes during 7-10 years, Stellato et al. (8) reported an increase in the risk for incident diabetes per s.D. decrease in free testosterone (OR 1.58, CI 1.08-2.29) in a multivariate model including BMI, while no predictive value of total testosterone was found. In a case-control study by Haffner et al. (19), a reduced age-adjusted risk for diabetes was reported for men having free but not total testosterone levels above the median (OR 0.63, CI 0.41-0.99), but the significance was lost in multivariate analyses including BMI. In 294 men of whom 26 developed diabetes over 8 years, Oh et al. (20) found an increased risk for diabetes when comparing the lowest quartile of total testosterone to the higher quartile (OR 2.7, CI 1.1-6.6) after multivariate adjustments including BMI, while bioavailable testosterone was not predictive for later diabetes. Chen et al. (21) found no association of total testosterone with incident diabetes in 195 men followed for 8 years, of whom eight developed diabetes, while not investigating the predictive value of free testosterone. Thus, this study together with other prospective reports does not provide a fully coherent picture as to the role of total and free testosterone levels in later diabetes risk. It also remains to be fully resolved whether the associations are independent of obesity or not.

The reduction in risk for later diabetes with higher SHBG levels, although not significant after adjustments for waist circumference in our analyses, is consistent with earlier prospective studies $(7,8,19)$, and in those observational studies examining the predictive role of both SHBG and testosterone, the risk estimates across quartiles are consequently stronger for SHBG than for free or total testosterone. Furthermore, a recent nested case-control study by Ding et al. (22) reported a strong reduction in risk for diabetes independent of BMI in men with SHBG levels in the highest quartile than in those with SHBG levels in the lowest quartile (HR 0.10, CI 0.03-0.36). Because reverse causation may be a concern (as prediabetic hyperinsulinemia may lower SHBG (23)), Ding et al. made a novel approach with similar findings after Mendelian randomization based on SHBG genotypes. The authors presented a strong support for a causative role of SHBG, and indicated a role for SHBG as a target in early risk stratification for diabetes.

In light of current evidence regarding the impact of SHBG on the prediction of later diabetes, one might question if earlier findings on the impact of total testosterone on later diabetes are confounded by SHBG, rather than being effects of total testosterone per se. Thus, we repeated the analyses intending to explore the independent contribution from total testosterone and SHBG when included in the same multivariate models. SHBG but not total testosterone was still independently predictive of subsequent diabetes when assessed linearly per s.D. increase after multivariate adjustments not including waist circumference (HR 0.58, CI 0.37-0.92). As SHBG and total testosterone are highly correlated $(r=0.63)$, there is a certain risk of colinearity affecting the result when including these two parameters in the same multivariate model, which is difficult to assess when Cox regression is used (as variance inflation factor is not an option like it is in linear regression). Furthermore, in one cross-sectional study of 2470 men by Yeap et al. (24), total testosterone but not SHBG was associated with insulin resistance. Nevertheless, recent findings on the role of SHBG in predicting diabetes, possibly to a stronger degree than total testosterone, highlight the need for randomized controlled trials with testosterone supplementation.

To date, few prospective studies have examined the impact of estrogens on later diabetes risk in men, and to our knowledge, this is the first prospective study to show an increased risk for diabetes in men with higher $E_{2}$ levels. Oh et al. (20) found no impact of bioavailable $\mathrm{E}_{2}$ on diabetes risk among 294 men from the Rancho Bernardo Study, of whom 26 got diabetes during 8 years of follow-up, and nor did Haffner et al. (19) report any impact of $E_{2}$ levels in a nested case-control study with 176 cases of diabetes. Although cross-sectional, Colangelo et al. reported a positive multi-adjusted association of $\mathrm{E}_{2}$ with diabetes among 3156 men from the Multi-Ethnic Study of Atherosclerosis (25), which is in accordance with our findings. No association between $\mathrm{E}_{2}$ and diabetes was reported by Selvin et al. among 1413 men from the Third National Health and Nutrition Examination Survey (26).

In men, Vermeulen et al. reviewed the relationship between age and various measures of obesity with male $E_{2}$ levels (27). In men, some $20 \%$ of the total $E_{2}$ is secreted by the testes, while the main source of $E_{2}$ is peripheral conversion of testosterone by aromatase. Levels of $E_{2}$ in males have been associated with various measures of obesity in most studies (27-31), though a few studies reported no association $(32,33)$, as was the case in the present study. Ten weeks of selective estrogen suppression did not lead to significant changes in body composition in a study of eight males who were 15-22 years of age (34). As the fat mass increases with age, the rate of aromatization of testosterone to $\mathrm{E}_{2}$ is believed to increase, but as there is an age-related decline in testosterone, the net effect of aging on male $\mathrm{E}_{2}$ levels is somewhat complex. Most studies have reported a slight decrease in $E_{2}$ levels with aging $(27,31,33,35)$, but an increase was also reported (29), as was the case in our analyses. In a study of 20 young male-to-female transsexuals, 1-year treatment with an estrogen and an anti-androgen led to an increase in fasting insulin with unchanged fasting glucose, a decrease in insulin sensitivity, and an increase in visceral and subcutaneous fat mass (36). In total, most studies including the present study support an unfavorable association of higher $\mathrm{E}_{2}$ levels in men with obesity, insulin resistance, and the prevalence and the incidence of diabetes. 
Strengths of this study are its prospective design, the large number of participants from a general population, the long-term follow-up, and the use of Cox regression in the analyses, allowing us to account for the time from baseline to a diagnosis of diabetes in the analyses. However, our study also has limitations, some of which are due to the fact that the Tromsø study was not initially set up to study the impact of sex hormones on later incidence of diabetes. Blood samples were drawn between 0800 and $1600 \mathrm{~h}$ and should ideally have been drawn in the morning as there is a diurnal variation in testosterone levels. However, the diurnal variation is less pronounced as men age (37), and our population had a mean age of 59 years. In addition, adjusting for sampling time in the analyses did not change the result. Blood samples were stored frozen for 7 years before they were first thawed for analysis in 2001. When stored frozen, levels of steroid hormones have been shown to be relatively stable for a period of up to 10 years $(38,39)$. Furthermore, a slight decrease in hormone levels would not be expected to alter the ordinal associations for the observed levels. The day-to-day variation in testosterone levels may be considerable, for which reason repeated tests are recommended for a diagnosis of hypogonadism. We did not account for the intraindividual day-to-day variation in our study in a single blood sample. Even if the ascertainment of possible diabetes cases was thorough, we may still have missed some cases since not all the participants of the fourth Tromsø study attended the sixth Tromsø study, from which possible cases were retrieved. Nevertheless, these are all sources of potential non-differential misclassification, tending to attenuate possible associations rather than produce spurious effects of sex hormones on later incidence of diabetes.

\section{Conclusion}

In conclusion, men with higher $E_{2}$ levels had an increased risk of later diabetes independent of obesity, while men with lower total testosterone and SHBG had an increased risk of diabetes that appeared to be dependent on obesity. This should encourage further research on the cause-effect relationship between sex hormones, SHBG, and diabetes in men.

\section{Declaration of interest}

The authors declare that there is no conflict of interest that could be perceived as prejudicing the impartiality of the research reported.

\section{Funding}

This work was supported by a grant (SFP729-08) from the Northern Norway Regional Health Authority.

\section{References}

1 Svartberg J, von Mühlen D, Sundsfjord J \& Jorde R. Waist circumference and testosterone levels in community dwelling men. The Tromsø study. European Journal of Epidemiology 200419 657-663.

2 Svartberg J, Von Mühlen D, Schirmer H, Barrett-Connor E, Sundfjord J \& Jorde R. Association of endogenous testosterone with blood pressure and left ventricular mass in men. The Tromsø study. European Journal of Endocrinology 2004150 65-71.

3 Haffner SM, Valdez RA, Mykkänen L, Stern MP \& Katz MS. Decreased testosterone and dehydroepiandrosterone sulfate concentrations are associated with increased insulin and glucose concentrations in nondiabetic men. Metabolism 199443 599-603.

4 Agledahl I, Skjærpe PA, Hansen JB \& Svartberg J. Low serum testosterone in men is inversely associated with non-fasting serum triglycerides: the Tromsø study. Nutrition, Metabolism, and Cardiovascular Diseases 200818 256-262.

5 Dhindsa S, Prabhakar S, Sethi M, Bandyopadhyay A, Chaudhuri A \& Dandona P. Frequent occurrence of hypogonadotropic hypogonadism in type 2 diabetes. Journal of Clinical Endocrinology and Metabolism 200489 5462-5468.

6 Svartberg J, Jenssen T, Sundsfjord J \& Jorde R. The associations of endogenous testosterone and sex hormone-binding globulin with glycosylated hemoglobin levels, in community dwelling men. The Tromsø study. Diabetes \& Metabolism 200430 29-34.

7 Laaksonen DE, Niskanen L, Punnonen K, Nyyssönen K, Tuomainen TP, Valkonen VP, Salonen R \& Salonen JT. Testosterone and sex hormone-binding globulin predict the metabolic syndrome and diabetes in middle-aged men. Diabetes Care 200427 1036-1041.

8 Stellato RK, Feldman HA, Hamdy O, Horton ES \& McKinlay JB. Testosterone, sex hormone-binding globulin, and the development of type 2 diabetes in middle-aged men: prospective results from the Massachusetts male aging study. Diabetes Care 200023 490-494.

9 Wang C, Swerdloff RS, Iranmanesh A, Dobs A, Snyder PJ, Cunningham G, Matsumoto AM, Weber T \& Berman N. Transdermal testosterone gel improves sexual function, mood, muscle strength, and body composition parameters in hypogonadal men. Journal of Clinical Endocrinology and Metabolism 200085 2839-2853.

10 Simon D, Charles MA, Lahlou N, Nahoul K, Oppert JM, GouaultHeilmann M, Lemort N, Thibult N, Joubert E, Balkau B \& Eschwege E. Androgen therapy improves insulin sensitivity and decreases leptin level in healthy adult men with low plasma total testosterone: a 3-month randomized placebo-controlled trial. Diabetes Care 200124 2149-2151.

11 Kapoor D, Goodwin E, Channer KS \& Jones TH. Testosterone replacement therapy improves insulin resistance, glycaemic control, visceral adiposity and hypercholesterolaemia in hypogonadal men with type 2 diabetes. European Journal of Endocrinology 2006154 899-906.

12 Boyanov MA, Boneva Z \& Christov VG. Testosterone supplementation in men with type 2 diabetes, visceral obesity and partial androgen deficiency. Aging Male 20036 1-7.

13 Yialamas MA, Dwyer AA, Hanley E, Lee H, Pitteloud N \& Hayes FJ. Acute sex steroid withdrawal reduces insulin sensitivity in healthy men with idiopathic hypogonadotropic hypogonadism. Journal of Clinical Endocrinology and Metabolism 200792 4254-4259.

14 Liu PY, Wishart SM, Celermajer DS, Jimenez M, Pierro ID, Conway AJ \& Handelman DJ. Do reproductive hormones modify insulin sensitivity and metabolism in older men? A randomized, placebo-controlled clinical trial of recombinant human chorionic gonadotropin European Journal of Endocrinology 2003148 55-66.

15 Pitteloud N, Mootha VK, Dwyer AA, Hardin M, Lee H, Eriksson KF, Tripathy D, Yialamas M, Groop L, Elahi D \& Hayes FJ. Relationship between testosterone levels, insulin sensitivity, and mitochondrial function in men. Diabetes Care 200528 1636-1642. 
16 Svartberg J, Midtby M, Bonaa KH, Sundsfjord J, Joakimsen RM \& Jorde R. The associations of age, lifestyle factors and chronic disease with testosterone in men: the Tromsø study. European Journal of Endocrinology 2003149 145-152.

17 Vermeulen A, Verdonck L \& Kaufman JM. A critical evaluation of simple methods for the estimation of free testosterone in serum. Journal of Clinical Endocrinology and Metabolism $1999 \mathbf{8 4}$ 3666-3672.

18 Mårin P, Holmäng S, Jönsson L, Sjöström L, Kvist H, Holm G, Lindstedt G \& Björntorp P. The effects of testosterone treatment on body composition and metabolism in middle-aged obese men. International Journal of Obesity 199216 991-997.

19 Haffner SM, Shaten J, Stern MP, Smith GD \& Kuller L. Low levels of sex hormone-binding globulin and testosterone predict the development of non-insulin-dependent diabetes mellitus in men. MRFIT Research Group. Multiple Risk Factor Intervention Trial. American Journal of Epidemiology 1996143 889-897.

20 Oh JY, Barrett-Connor E, Wedick NM \& Wingard DL. Endogenous sex hormones and the development of type 2 diabetes in older men and women: the Rancho Bernardo study. Diabetes Care 200225 55-60.

21 Chen RY, Wittert GA \& Andrews GR. Relative androgen deficiency in relation to obesity and metabolic status in older men. Diabetes, Obesity \& Metabolism 20068 429-435.

22 Ding EL, Song Y, Manson JE, Hunter DJ, Lee CC, Rifai N, Buring JE, Gaziano JM \& Liu S. Sex hormone-binding globulin and risk of type 2 diabetes in women and men. New England Journal of Medicine 2009361 1152-1163.

23 Kalme T, Koistinen H, Loukovaara M, Koistinen R \& Leinonen P. Comparative studies on the regulation of insulin-like growth factor-binding protein-1 (IGFBP-1) and sex hormone-binding globulin (SHBG) production by insulin and insulin-like growth factors in human hepatoma cells. Journal of Steroid Biochemistry and Molecular Biology 200386 197-200.

24 Yeap BB, Chubb SA, Hyde Z, Jamrozik K, Hankey GJ, Flicker L \& Norman PE. Lower serum testosterone is independently associated with insulin resistance in non-diabetic older men: the Health in Men Study. European Journal of Endocrinology 2009161 591-598.

25 Colangelo LA, Ouyang P, Liu K, Kopp P, Golden SH, Dobs AS, Szklo M, Vaidya D, Cushman M \& Gapstur SM. Association of endogenous sex hormones with diabetes and impaired fasting glucose in men: Multi-Ethnic Study of Atherosclerosis. Diabetes Care 200932 1049-1051.

26 Selvin E, Feinleib M, Zhang L, Rohrmann S, Rifai N, Nelson WG, Dobs A, Basaria S, Golden SH \& Platz EA. Androgens and diabetes in men: results from the Third National Health and Nutrition Examination Survey (NHANES III). Diabetes Care 200730 234-238.

27 Vermeulen A, Kaufman JM, Goemaere S \& van Pottelberg I. Estradiol in elderly men. Aging Male 20025 98-102.
28 Muller M, den Tonkelaar I, Thijssen JH, Grobbee DE \& van der Schouw YT. Endogenous sex hormones in men aged 40-80 years. European Journal of Endocrinology 2003149 583-589.

29 Gyllenborg J, Rasmussen SL, Borch-Johnsen K, Heitmann BL, Skakkebaek NE \& Juul A. Cardiovascular risk factors in men: the role of gonadal steroids and sex hormone-binding globulin. Metabolism $2001 \mathbf{5 0} 882-888$.

30 Jensen TK, Andersson AM, Jørgensen N, Andersen AG, Carlsen E, Petersen JH \& Skakkebaek NE. Body mass index in relation to semen quality and reproductive hormones among 1,558 Danish men. Fertility and Sterility $2004 \mathbf{8 2} 863-870$.

31 Phillips GB. Relationship between serum sex hormones and the glucose-insulin-lipid defect in men with obesity. Metabolism 1993 42 116-120.

32 Phillips GB, Jing T \& Hevmsfield SB. Relationships in men of sex hormones, insulin, adiposity, and risk factors for myocardial infarction. Metabolism 200352 784-790.

33 van den Beld AW, de Jong FH, Grobbee DE, Pols HA \& Lamberts SW. Measures of bioavailable serum testosterone and estradiol and their relationships with muscle strength, bone density, and body composition in elderly men. Journal of Clinical Endocrinology and Metabolism 200085 3276-3282.

34 Mauras N, O'Brien KO, Klein KO \& Hayes V. Estrogen suppression in males: metabolic effects. Journal of Clinical Endocrinology and Metabolism 200085 2370-2377.

35 Leifke E, Gorenoi V, Wichers C, Von Zur Mühlen A, Von Büren E \& Brabant G. Age-related changes of serum sex hormones, insulinlike growth factor-1 and sex-hormone binding globulin levels in men: cross-sectional data from a healthy male cohort. Clinical Endocrinology 200053 689-695.

36 Elbers JM, Giltay EJ, Teerlink T, Scheffer PG, Asscheman H, Seidell JC \& Gooren LJ. Effects of sex steroids on components of the insulin resistance syndrome in transsexual subjects. Clinical Endocrinology 200358 562-571.

37 Bremner WJ, Vitiello MV \& Prinz PN. Loss of circadian rhythmicity in blood testosterone levels with aging in normal men. Journal of Clinical Endocrinology and Metabolism 198356 1278-1281.

38 Kley HK, Schlaghecke R \& Krüskemper HL. Stability of steroids in plasma over a 10-year period. Journal of Clinical Chemistry and Clinical Biochemistry 198523 875-878.

39 Bolelli G, Muti P, Micheli A, Sciajno R, Franceschetti F, Krogh V, Pisani P \& Berrino F. Validity for epidemiological studies of longterm cryoconservation of steroid and protein hormones in serum and plasma. Cancer Epidemiology Biomarkers \& Prevention 19954 509-513.

Received 30 November 2009

Accepted 29 December 2009 